\title{
Economic Valuation of the Global Burden of Cleft Disease Averted by a Large Cleft Charity
}

\author{
Dan Poenaru ${ }^{1} \cdot$ Dan $\mathrm{Lin}^{2} \cdot$ Scott Corlew
}

Published online: 15 December 2015

(C) Société Internationale de Chirurgie 2015

\begin{abstract}
Background This study attempts to quantify the burden of disease averted through the global surgical work of a large cleft charity, and estimate the economic impact of this effort over a 10-year period.

Methods Anonymized data of all primary cleft lip and cleft palate procedures in the Smile Train database were analyzed and disability-adjusted life years (DALYs) calculated using country-specific life expectancy tables, established disability weights, and estimated success of surgery and residual disability probabilities; multiple age weighting and discounting permutations were included. Averted DALYs were calculated and gross national income (GNI) per capita was then multiplied by averted DALYs to estimate economic gains.

Results 548,147 primary cleft procedures were performed in 83 countries between 2001 and 2011. 547,769 records contained complete data available for the study; $58 \%$ were cleft lip and $42 \%$ cleft palate. Averted DALYs ranged between 1.46 and $4.95 \mathrm{M}$. The mean economic impact ranged between USD 5510 and 50,634 per person. This corresponded to a global economic impact of between USD 3.0B and 27.7B USD, depending on the DALY and GNI values used. The estimated cost of providing these procedures based on an average reimbursement rate was USD $197 \mathrm{M}(0.7-6.6 \%$ of the estimated impact).

Conclusions The immense economic gain realized through procedures focused on a small proportion of the surgical burden of disease highlights the importance and cost-effectiveness of surgical treatment globally. This methodology can be applied to evaluate interventions for other conditions, and for evidence-based health care resource allocation.
\end{abstract}

\section{Introduction}

Several metrics have been used in the measurement of health interventions, including life expectancy, mortality rates, disability-adjusted life years (DALYs), quality-

Scott Corlew

dscorlew@gmail.com

1 MyungSung Christian Medical Center, Addis Ababa, Ethiopia and Montreal Children's Hospital, Montreal, Canada

2 Rollins School of Public Health, Emory University, Atlanta, GA, USA

32111 Riverview Drive, Murfreesboro, TN 37129, USA adjusted life years (QALYs), and others [1-3]. However, in order to compare the societal impact of health care to other sectors, a common measurement tool is needed. Economic assessment is such a cross-sector "universal currency," its main drawback being the difficulty of measuring economic benefit.

This study uses a framework derived from previous work [4-11] to examine the economic value of the programs of a non-governmental organization. Smile Train (ST) supports the surgical care of patients with cleft lip and palate (CLP) in low- and middle-income countries (LMICs). ST identifies and trains surgical teams in each country and contracts to provide operative care for a predetermined fee per case [12]. This model is akin to the 


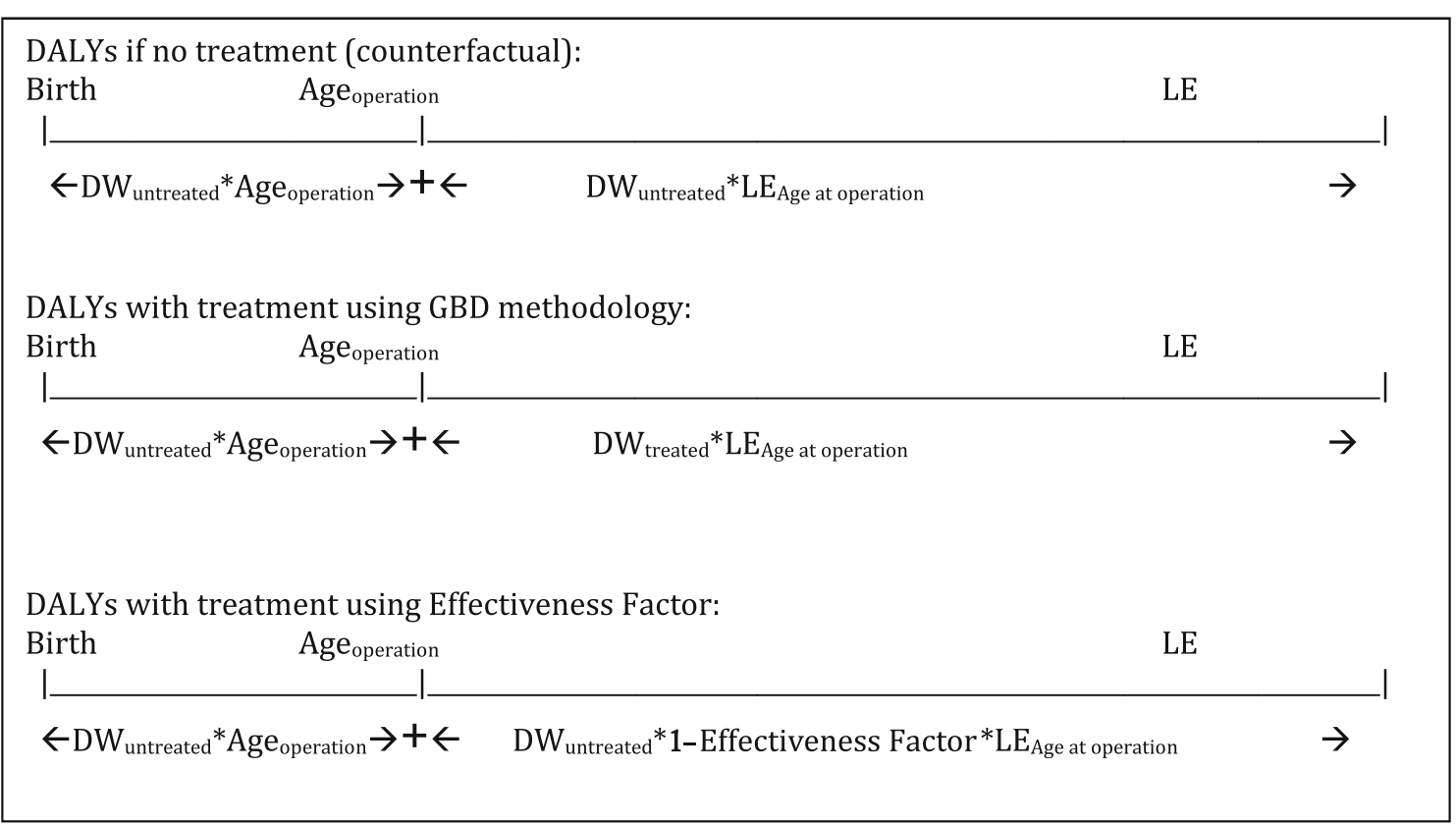

Fig. 1 Diagram of age milestones and calculations used for estimating averted DALYs

specialty surgical hospital platform described by Shrime et al. [13], but uses LMIC surgeons exclusively. Given that CLP impacts the social, physical, and economic lives of affected individuals, this study estimates the economic impact of the ST work, providing a framework for examining the value of health interventions for cross-sector comparison.

\section{Methods}

The approach was to estimate the economic productivity of treated individuals, then derive the counterfactual of their economic productivity had they not been beneficiaries of the programs. The difference between these two figures, is presented as the economic effect of the program. The difference in DALYs is also presented.

The database of all procedures performed in ST programs between 2001 and 2011 was utilized. Only primary (first-time) CLP repair procedures were included. Countryspecific life expectancy (LE) values were used, rather than a universal value as used by the Global Burden of Disease (GBD) study [14].

The standard DALY formula was used for burden of disease $(\mathrm{BoD})$ : DALYs $=$ YLL (Years of Life Lost $)+$ YLD (Years Lived with Disability). The YLL factor was omitted in light of the small mortality of cleft lip and palate.

The counterfactual BoD in DALYs potentially incurred by each patient without the surgical intervention (which includes the non-avertable as well as the avertable DALYs [15]), was estimated thus:

$$
\begin{aligned}
\mathrm{BoD}_{\text {without intervention }}= & \left(\mathrm{DW}_{\text {untreated }} * \text { age }_{\text {operation }}\right) \\
& +\left(\mathrm{DW}_{\text {untreated }} * \mathrm{LE}_{\text {age at treatment }}\right),
\end{aligned}
$$

where $\mathrm{DW}_{\text {untreated }}=$ disability weight for CLP untreated (both $\mathrm{DW}_{\text {untreated }}$ and $\mathrm{DW}_{\text {treated }}$ from the GBD study [16] and $\mathrm{LE}_{\text {age }}$ at treatment $=$ life expectancy at the age the operation occurred, from the Standard West Level 26 Life Table [17]).

DALYs actually incurred by each patient, or the

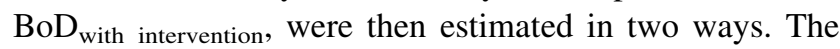
first used the GBD study method for calculating DALYs:

$$
\begin{aligned}
\mathrm{BoD}_{\text {with intervention }}= & \left(\mathrm{DW}_{\text {untreated }} * \text { age }_{\text {operation }}\right) \\
& +\left(\mathrm{DW}_{\text {treated }} * \mathrm{LE}_{\text {age at operation }}\right)
\end{aligned}
$$

DALYs incurred with the intervention were also estimated by the method published by McCord et al. [11] and widely followed in the surgical literature [18-21]. This method multiplies the ideal impact of surgical intervention by the risk of permanent disability without surgery (RPD) and the estimate of residual disability (ERD) after the operation. The latter factor aims to account for the residual effects of the deformity, the possibility of post-operative complications, and the possible need for further procedures. In CLP, the RPD is 1.0, since disability is expected in the absence of surgery in $100 \%$ of cases, while the resolution of disability (1-ERD) was estimated to be 
between 75 and $94 \%$ for cleft lip and between 25 and $74 \%$ for cleft palate. For the purpose of this study, the two factors were combined into a single "Effectiveness Factor" (EF) of 0.8 for cleft lip and 0.5 for cleft palate, similar to the previously used "probability of successful treatment" (PST) for CLP [10]. This Effectiveness Factor postulates that the operation, on average, resulted in resolution of $80 \%$ of the residual disability for cleft lip and $50 \%$ for cleft palate patients. This EF was hence used in place of the $\mathrm{DW}_{\text {treated: }}$

$$
\begin{aligned}
\mathrm{BoD}_{\text {with intervention }}= & \left(\mathrm{DW}_{\text {untreated }} * \mathrm{Age}_{\text {operation }}\right) \\
& +\left(\mathrm{DW}_{\text {untreated }} *(1-\mathrm{EF})\right. \\
& \left.* \mathrm{LE}_{\text {Age at operation }}\right)
\end{aligned}
$$

The difference between DALYs without and with surgical intervention represents the effect of the intervention on the health status of the patient and, globally, on alleviating BoD. Figure 1 shows the calculation markers in a schematic fashion.

DALYs were calculated with and without age weighting as was done in the original GBD study [22], and with and without $3 \%$ future discounting.

These averted DALY values were then multiplied by the GNI per capita for each country [23] to give the economic value added to the national economy over the lifetime of each patient. Using both the Atlas and Purchasing Power Parity (PPP) methods, two economic totals for each country were derived for each DALY calculation. The methodologies used in this study for calculating DALYs and the economic gain are shown diagrammatically in Fig. 2.

Individual totals were summed to give an estimate of economic value added for the entire program. This was reported by year and for the entire period of the study.

A cost-effectiveness analysis (CEA) was performed as previously reported for Smile Train [10]. As actual costs at the hundreds of individual sites vary widely and were not available, the set contributions per procedure paid by the organization were used as a proxy.

Data analysis and simple descriptive statistics were performed in Microsoft Excel ${ }^{\circledR}$.

\section{Results}

Tables 1 and 2 show the results in very accessible format. Between 2001 and 2011, 548,147 primary operations to repair CLP were performed in 83 countries. Figure 3 shows the cases available for study and the patient characteristics.

Total averted DALYs achieved by method of calculation and year are shown in Fig. 4. Table 1 details the DALYs averted by procedure and by the various calculation methods. Using the GBD DW method, about one-third of the averted BoD was due to cleft lip repairs and two-thirds to cleft palate repairs; using the effectiveness factor method, this was slightly greater than half, both reflecting the greater disability associated with cleft palate.

Values derived for the economic gain from repair of cleft lip and palate using the Atlas methodology for GNI ranged between 3.0 billion and 10.7 billion USD and between 7.9 billion and 27.7 billion USD using PPP. Using the effectiveness factor, for each person undergoing cleft lip repair, the average economic gain, calculated without age weighting or discounting, was 9907 USD using the Atlas method and 26,426 USD using PPP. For cleft palate, these gains were 17,227 USD by Atlas and 44,064 using PPP. Using the GBD method of calculating DALYs, these figures were 10,362 USD Atlas and 27,639 USD PPP for cleft lip and 32,216 USD Atlas and 82,405 USD PPP for cleft palate. These data are detailed in Table 2. Figure 5 depicts the ranges of economic impact by method per year.

The overall cost for the interventions studied was estimated at 197 million USD, which represents between 0.7 and $6.6 \%$ of the estimated economic gain, depending on the valuation method used. In terms of cost-effectiveness, this represents between $\$ 40$ and 135/DALY.

\section{Discussion}

This study is an effort to quantify the value of a global surgical program in economic terms. Besides providing economic data on the impact of surgical repair of CLP, the methodology lends itself to similar calculations in other specialties and programs.

The current study builds on several preceding reports of economic modeling of interventions [4, 6, 8, 9, 24]. Methodologically, measuring the economic benefit of a health intervention requires four data points: definition of the health problem, definition of the intervention and its probability of success, quantitative estimates of the change due to the intervention, and a method of converting the health effect into economic terms [5]. All of these require significant assumptions and are subject to multiple methodological approaches to address the assumptions. These lead to wide confidence intervals, but barring extensive, expensive, and lengthy direct studies of individual economic productivity, such assumptions are necessary.

While the anatomic clefts are the core problems comprising the defects studied, there are secondary issues: hearing problems, increased rates of infection, difficulties eating and corresponding malnutrition, and orthodontic problems. These are addressed through the DWs from the GBD study and the "effectiveness factor" (EF) method. 


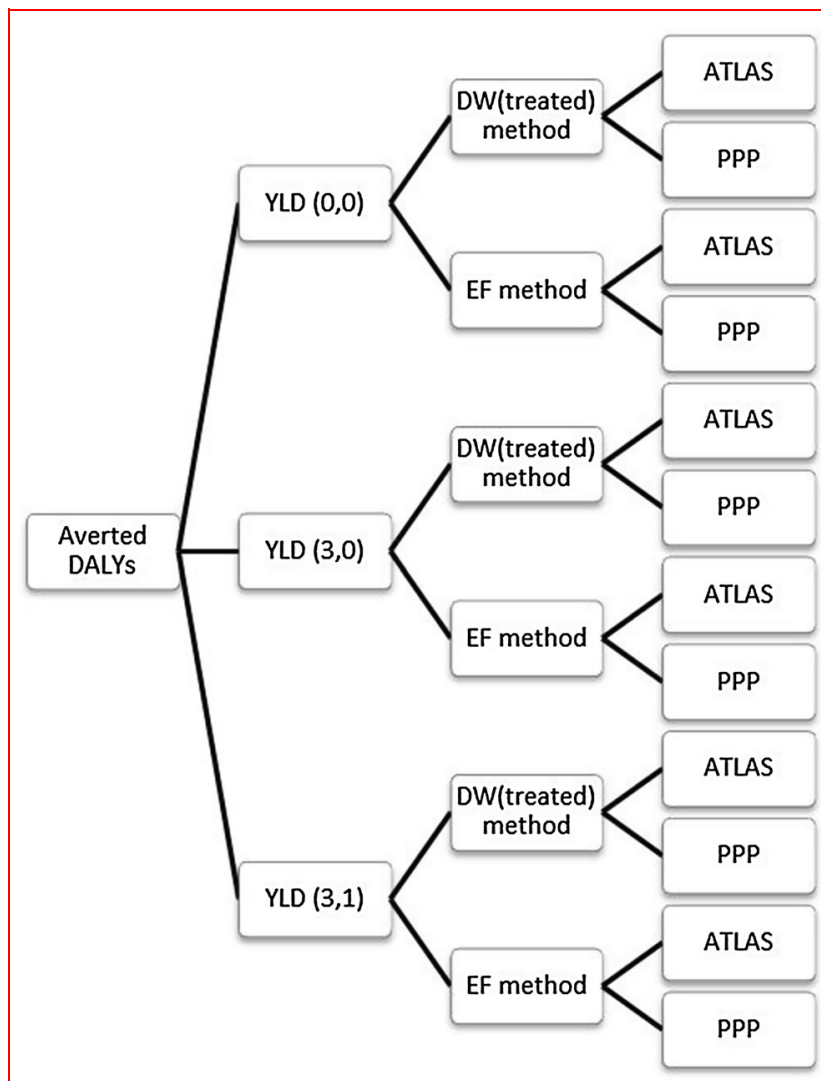

Fig. 2 Diagram of sensitivity analysis methodologies used in calculating the economic value of interventions
In regard to the interventions, the mean age of the patients was quite advanced, reflecting a significant back$\log [10]$ and delayed access to surgical care in LMICs. The preponderance of cleft lip repairs does not reflect the expected relative incidence of CLP, thus pointing towards a possible tendency to repair cleft lips but not palates. This has been observed in low-resource settings [10] and may even reflect a hidden mortality of cleft palate infants [25]. Finally, the gender distribution is skewed with a preponderance of males, a potential reflection on gender inequity issues in many low-resource settings.

The DALY data reflect the large $\mathrm{BoD}$ which is avertable through surgical intervention even in a narrow specialty. The 1-5 million DALYs averted over 10 years are viewed in the context of an estimated 25 million DALYs for overall surgical BoD in Africa [26], and the mean averted DALYs per patient are similar to those estimated for hydrocephalus and inguinal hernia [6, 27].

The economic value of improved health resulting from the surgical interventions was determined using GNI per capita, based on the premise that each individual theoretically contributed an equal share. A first assumption was that CLP affects equally all sectors of society regardless of socioeconomic stratum, a reasonable assumption based on current epidemiologic knowledge. A second assumption was that DALYs account for all the social, psychological, and secondary losses associated with CLP. Alternative econometric methodologies such as labor productivity,

Table 1 Averted burden of disease in DALYs per patient and total

\begin{tabular}{|c|c|c|c|}
\hline & 0.0 & 3.0 & 3.1 \\
\hline \multicolumn{4}{|c|}{ Sum of averted DALYs using Eff factor } \\
\hline Cleft palate & $1,749,252$ & 759,125 & 921,451 \\
\hline Cleft lip & $1,607,513$ & 704,900 & 834,406 \\
\hline Total & $3,356,765$ & $1,464,025$ & $1,755,857$ \\
\hline \multicolumn{4}{|c|}{ Sum of averted DALYs using GBD DW treated versus untreated } \\
\hline Cleft palate & $3,271,329$ & $1,419,662$ & $1,723,233$ \\
\hline Cleft lip & $1,681,327$ & 737,268 & 872,721 \\
\hline Total & $4,952,656$ & $2,156,930$ & $2,595,954$ \\
\hline \multicolumn{4}{|c|}{ Average of averted DALYs per pt using Eff factor } \\
\hline Cleft palate & 7.61 & 3.30 & 4.01 \\
\hline Cleft lip & 5.06 & 2.22 & 2.63 \\
\hline Total & 6.13 & 2.67 & 3.21 \\
\hline \multicolumn{4}{|c|}{ Average of averted DALYs per pt using GBD DW treated versus untreated } \\
\hline Cleft palate & 14.22 & 6.17 & 7.49 \\
\hline Cleft lip & 5.29 & 2.32 & 2.75 \\
\hline Total & 9.04 & 3.94 & 4.74 \\
\hline
\end{tabular}


Table 2 Economic gain per patient and total by method of calculation

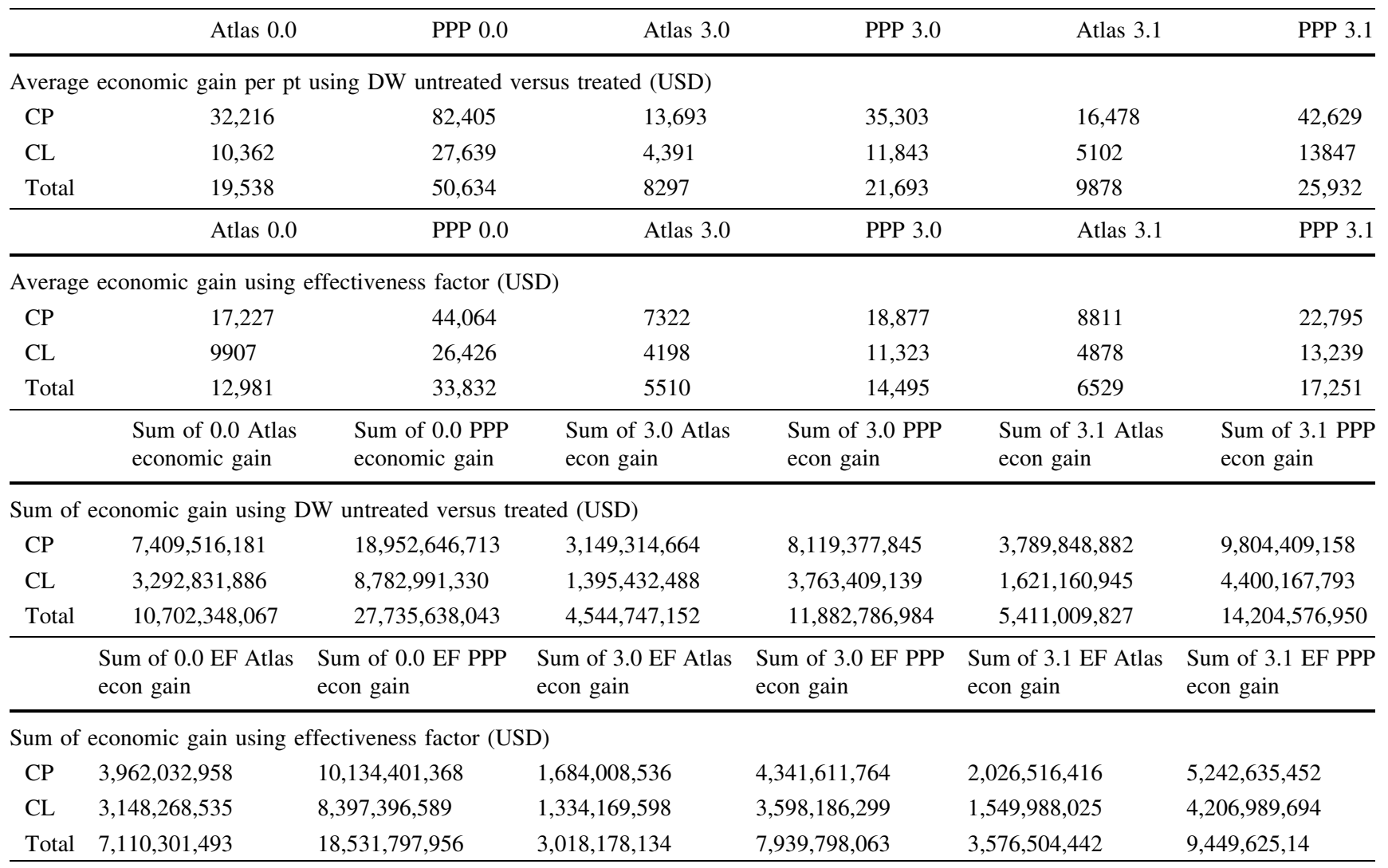

Fig. 3 Cases available for study and Patient characteristics

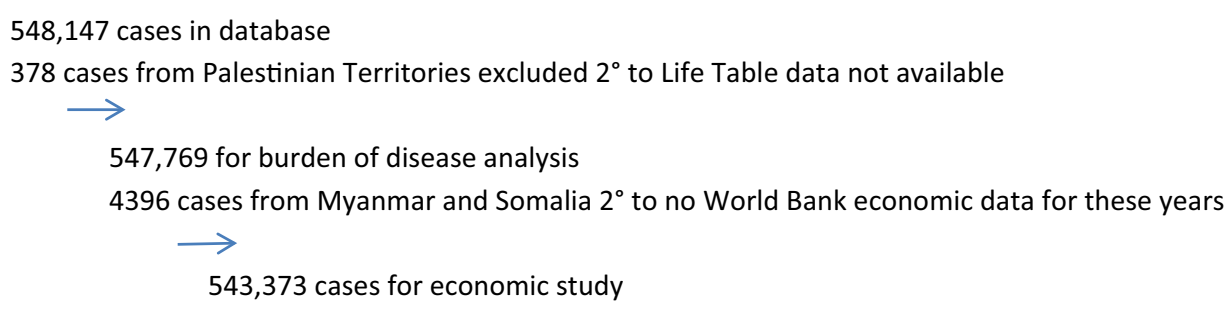

378 cases from Palestinian Territories excluded $2^{\circ}$ to Life Table data not available $\longrightarrow$

547,769 for burden of disease analysis

4396 cases from Myanmar and Somalia $2^{\circ}$ to no World Bank economic data for these years

543,373 cases for economic study

No PPP data for Argentina, and Atlas data only through 2006, so Argentina cases after 2006 used 2006 GNI/cap data; Argentina cases excluded from PPP analysis.

Djibouti data only available through 2005, so Djibouti cases after 2005 used 2005 GNI/cap data

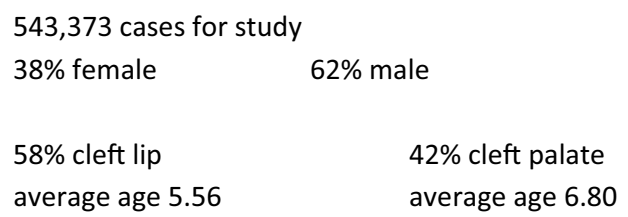

willingness to pay, value of a statistical life, or direct income studies may be the focus of future studies.

The GNI data indicate a very substantial economic impact, in keeping with other reports on surgery in limited resource settings. Using a similar methodology, Alkire et al. estimated the economic impact of treating CLP in
Sub-Saharan Africa (SSA) to USD 252-441 million, while Warf et al. projected the economic impact of surgically treating hydrocephalus in SSA to around 1 billion USD [6].

Cost-effectiveness analysis (CEA) is significantly limited in this context by the assumptions necessary in the modeling, but is a natural extension of studies of economic 


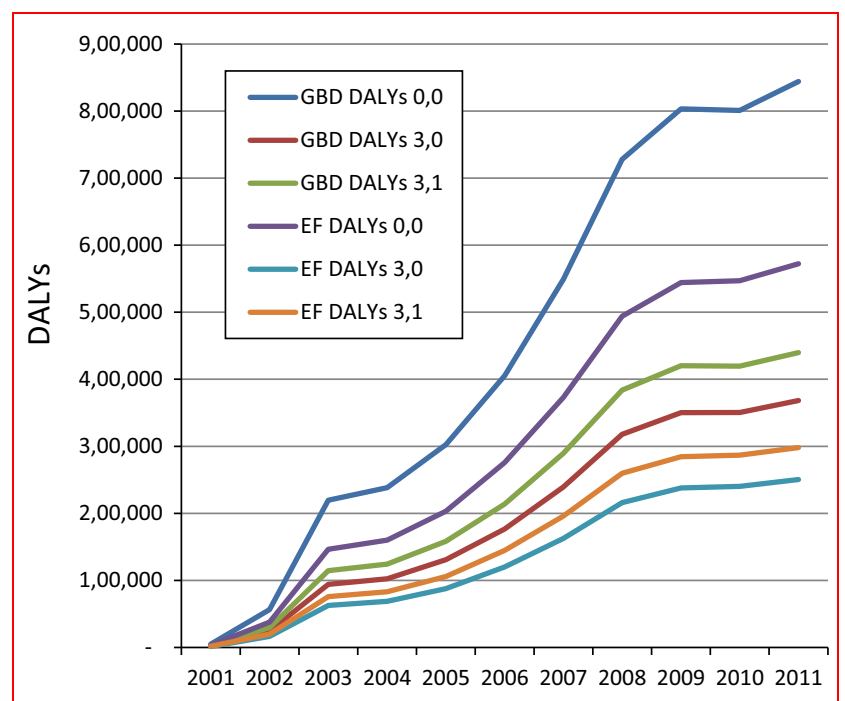

Fig. 4 Averted BoD through cleft lip and palate surgery by method and year

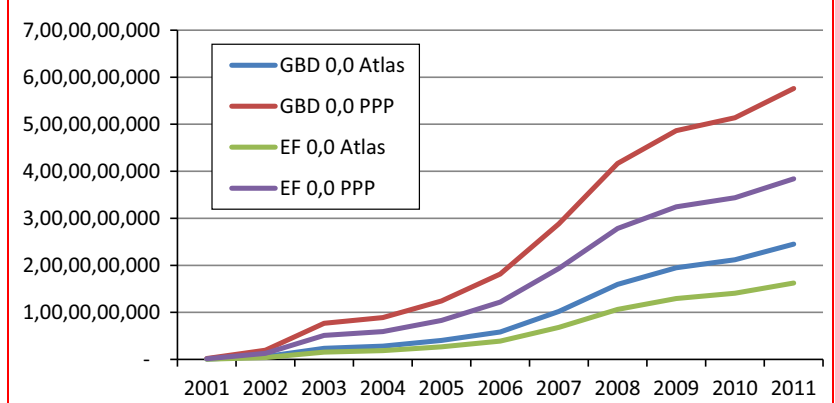

Fig. 5 Economic impact by year and calculation method without age weighting or discounting

impact. A frequent metric of CEA is the \$/DALY. The current estimate of \$40-135/DALY compares favorably with a previous report from Smile Train of \$70-134/DALY for CLP repairs [10] and with other CLP CEA studies ranging between USD 29-285/DALY [4, 28, 29].

\section{Limitations}

There are several limitations to the current study, some of which have been alluded to above. First, the sample is not random - the locale of the interventions, both nationally and regionally, reflects strategic and logistic choices within one large non-governmental organization. Absent data for some countries compound this effect, though it affects a very small proportion of the entire dataset.

DALY calculations rely on DWs which are notoriously difficult to estimate accurately [30] and on subjective effectiveness factors. In the face of multiple competing strategies such as age weighting and future discounting, the authors' only recourse was to offer ranges rather than precise values. One specific additional limitation of our study was the assumption that mortality from CLP is negligible, which in some low-resource settings may not be true [25]. Higashi et al. found that in addition to a small mortality attributable to the cleft deformity, there also was a higher all-cause mortality in unrepaired than repaired clefts throughout life [31]. Any mortality associated with cleft deformities would serve to increase our estimates of economic benefit to repair. The uncertainty is also exacerbated by the econometric estimates, which include two alternative methodologies (Atlas and PPP) yielding disparate results.

This study does not purport to evaluate complications, quality of care, externalities, or any other measures of the specific procedures performed. It also does not specifically account for the costs of the procedures; our cost-effectiveness analysis relied on the average reimbursement rates paid by the organization for each intervention.

There are other reasons for believing that our economic estimates, while impressively high, are still underestimates. It is well-recognized that school attendance, cognitive development, and social development during childhood have significant effects on adult productivity [32], and this is not included in the DALY estimates. The Smile Train work also includes a number of secondary procedures which increase the economic impact of this surgical program.

\section{Conclusions}

The current study documents the tremendous economic value of a scaled surgical program. Whether the actual contribution to the global economy is closer to 3 billion or 30 billion USD, it highlights the significant economic and public health value of vertical surgical interventions like CLP repair in low-resource settings. For a donor cost under 200 million USD, a 15- to 150-fold increase was added to the economies of the 83 beneficiary countries. Moreover, the clinical effect of these surgical interventions is a permanent one, not requiring ongoing therapy or expense to maintain or renew the gain. Congenital anomalies such as CLP are the ultimate "poverty trap" as described by Banerjee and Duflo [33], as without resolution of these issues, there is little opportunity for the individual who otherwise has the same potential as any other person to break out of her/his economic state.

While the current study is limited to a small area of surgical care, similar studies can be undertaken in other specialties. Such studies would add to the growing body of evidence supporting the value and cost-effectiveness of 
surgical care as a primary health intervention globally. This should inform advocacy efforts for resource allocation in the funding of health care globally.

Acknowledgments The authors express their appreciation to MacKinnon Engen of The Smile Train for making the database available and facilitating our analysis.

\section{References}

1. Lopez AD, Mathers CD, Ezzati M, Jamison DT, Murray CJL (2006) Global burden of disease and risk factors. Oxford University Press and The World Bank

2. Murray CJL, Salomon JA, Mathers C (2000) A critical examination of summary measures of population health. Bull WHO 78(8):981-994

3. Gold MR, Stevenson D, Fryback DG (2002) HALYS AND QALYS AND DALYS, OHMY: similarities and differences in summary measures of population. HealthAnnu Rev Public Health 23:115-134

4. Corlew DS (2010) Estimation of impact of surgical disease through economic modeling of cleft lip and palate care. World $\mathrm{J}$ Surg 34(3):391-396

5. Corlew D (2013) Economic modeling of surgical disease: a measure of public health interventions. World J Surg 37:1478-1485. doi:10.1007/s00268-012-1796-5

6. Warf BC, Alkire BC, Bhai S, Hughes C, Schiff SJ, Vincent JR, Meara JG (2011) Costs and benefits of neurosurgical intervention for infant hydrocephalus in sub-Saharan Africa. J Neurosurg Pediatr 8(5):509-521

7. Tan-Torres Edejer $\mathrm{T}$, Baltussen R, Adam $\mathrm{T}$, Hutubessy $\mathrm{R}$, Acharya A, Evans DB, Murray CJL, eds (2003). WHO Guide to cost-effectiveness analysis. Geneva, pp 1-239

8. Alkire B, Hughes CD, Nash K, Vincent JR, Meara JG (2011) Potential economic benefit of cleft lip and palate repair in subSaharan Africa. World J Surg 35(6):1194-1201. doi:10.1007/ s00268-011-1055-1

9. Alkire BC, Vincent JR, Burns CT, Metzler IS, Farmer PE, Meara JG (2012) Obstructed labor and caesarean delivery: the cost and benefit of surgical intervention. PLoS ONE 7(4):e34595

10. Poenaru D (2013) Getting the Job Done: analysis of the impact and effectiveness of the smiletrain program in alleviating the global burden of cleft disease. World J Surg 37(7):1562-1570. doi:10.1007/s00268-012-1876-6

11. McCord C, Chowdhury Q (2003) A cost effective small hospital in Bangladesh: what it can mean for emergency obstetric care. Int J Gynaecol Obstet 81(1):83-92

12. Smile Train: leading children's charity. www.smiletrain.org. Accessed March 31, 2014

13. Shrime MG, Sleemi A, Ravilla TD (2015) Charitable platforms in global surgery: a systematic review of their effectiveness, costeffectiveness, sustainability, and role training. World J Surg 39:10-20. doi:10.1007/s00268-014-2516-0

14. Murray CJL, Lopez A (1996) A comprehensive assessment of mortality and disability from disease, injures and risk factors in 1990 and projected to 2020. In The global burden of disease. Harvard University Press, Cambridge, pp 1-51

15. Bickler S, Ozgediz D, Gosselin R, Weiser T, Spiegel D, Hsia R, Dunbar P, McQueen K, Jamison D (2010) Key concepts for estimating the burden of surgical conditions and the unmet need for surgical care. World J Surg 34(3):374-380. doi:10.1007/ s00268-009-0261-6

16. Lopez A (2006) Global burden of disease and risk factors. (Lopez AD, Mathers CD, Ezzati M, Jamison DT, Murray CJL, Eds. Oxford University Press \& The World Bank, Library. New York

17. World Health Organization (2015) Standard Life Tables. Who.int/healthinfo/bodreferencesstandardlifetable.xls. Accessed Jan 21

18. Shillcutt SD, Clarke MG, Kingsnorth AN (2010) Cost-effectiveness of groin hernia surgery in the Western Region of Ghana. Arch Surg (Chicago, IL: 1960) 145(10):954-961

19. Gosselin RA, Maldonado A, Elder G (2010) Comparative costeffectiveness analysis of two MSF surgical trauma centers. World J Surg 34(3):415-419. doi:10.1007/s00268-009-0230-0

20. Gosselin RA, Thind A, Bellardinelli A (2006) Cost/DALY averted in a small hospital in Sierra Leone: what is the relative contribution of different services? World J Surg 30(4):505-511. doi:10.1007/s00268-005-0609-5

21. Wu VK, Poenaru D (2013) Burden of surgically correctable disabilities among children in the Dadaab Refugee Camp. World J Surg 37(7):1536-1543. doi:10.1007/s00268-012-1899-z

22. Murray CJL, Lopez AD, Jamison DT (1994) The global burden of disease in 1990: summary results, sensitivity analysis and future direction. Bull WHO 72(3):495-509

23. World Bank website: http://databank.worldbank.org/data/views/ reports/tableview.aspx. Accessed Jan 21, 2015

24. Hughes CD et al (2012) The Clinical and economic impact of a sustained program in global plastic surgery: valuing cleft care in resource-poor settings. PRS. doi:10.1097/PRS.0b013e3182 $54 \mathrm{~b} 2 \mathrm{a} 2$

25. Wilson J, Hodges A (2011) Cleft lip and palate surgery carried out by one team in Uganda: where have all the palates gone? Cleft Palate Craniofac J 49(3):1-7

26. Ozgediz D, Riviello R (2008) The "other" neglected diseases in global public health: surgical conditions in sub-Saharan Africa. PLoS Med 5(6):e121

27. Shillcutt SD, Sanders DL, Teresa Butrón-Vila M, Kingsnorth AN (2013) Cost-effectiveness of inguinal hernia surgery in northwestern Ecuador. World J Surg 37(1):32-41. doi:10.1007/ s00268-012-1808-5

28. Alkire B, Vincent J, Meara J (2014) Benefit-cost analysis of a cleft lip and palate surgical subspecialty hospital in India. In DCP3 draft, pp 1-17

29. Moon W, Perry H, Baek R-M (2012) Is international volunteer surgery for cleft lip and cleft palate a cost-effective and justifiable intervention? A case study from east Asia. World J Surg 36:2819-2830. doi:10.1007/s00268-012-1761-3

30. Gosselin R, Ozgediz D, Poenaru D (2013) A square peg in a round hole? challenges with DALY-based "burden of disease" calculations in surgery and a call for alternative metrics. World J Surg 37(11):2507-2511. doi:10.1007/s00268-013-2182-7

31. Higashi H, Barendregt JJ, Kassebaum NJ, Weiser TG, Bickler SW, Vos T (2015) The burden of selected congenital anomalies amenable to surgery in low and middle-income regions: cleft lip and palate, congenital heart anomalies and neural tube defects. Arch Dis Child 100:233-238

32. Bloom DE and Canning D (2008), Population health and economic growth, Working paper 24, Commission on Growth and Development, The World Bank

33. Banerjee AV, Duflo E (2012) Poor economics: a radical rethinking of the way to fight global poverty, Public Affairs 